Mars, 1998

\title{
ABSTRACT CONVEXITY, SOME RELATIONS AND APPLICATIONS ${ }^{1}$
}

\author{
Juan Vicente LLINARES ${ }^{2}$
}

$\mathrm{N}^{\circ} 9803$

\footnotetext{
${ }^{1}$ This research has been supported by a TMR scholarship of the EU under contract ERBFMBICT 961591

${ }^{2}$ I would like to express my gratitude to M. Florenzano, Ch. D. Horvath, J.E. Martínez Legaz, J.E. Peris, M.C. Sánchez and B. Subiza for their helpful comments.

CEPREMAP, 140 rue de Chevaleret, 75013 Paris (France)
} 


\title{
CONVEXITÉ ABSTRAITE, APPLICATIONS ET RELATIONS.
}

\author{
Résumé.
}

Le but de ce papier est d'étudier la relation entre différentes structures de convexité abstraite qui ont été utilisées pour étendre des résultats de point fixes et de sélection des correspondances, [convexité simpliciale (Bielawski, 1987), c-espace (Horvath 1987, 1991), order-convexité (Horvath et Llinares, 1996), B-convexité simpliciale, L-convexité (Ben-El-Mechaiekh et al., 1997), et mc-espaces (Llinares, 1997)].

Dans le contexte des mc-espaces, on démontre un résultat de caractérisation du problème d'intersection finie du type KnasterKuratowski-Mazurkiewicz, quelques conséquences et quelque généralisations du résultat de Browder d'existence d'une sélection continue et d'un point fixe pour les applications multivoques à fibres ouvertes.

Mots clés: Convexité abstraite, point fixe, KKM.

\section{ABSTRACT CONVEXITY, SOME RELATIONS AND APPLICATIONS.}

\begin{abstract}
.
The aim of this paper is to analyze the relationship between different notions of abstract convexity structures that we can find in the literature in connection with the problem of the existence of continuous selections and fixed points to correspondences. Mainly we will focus in the notion of mcspaces, which was introduced in LLinares $(1994,1997)$, and its relationship with c-spaces (Horvath, 1991), simplicial convexity (Bielawski, 1987), an order convexity (used in Horvath and Llinares, 1996), B'-simplicial convexity and L-spaces (Ben-El-Mechaiekh et.al., 1997). Moreover, in the context of mc-spaces, a characterization result of non-empty finite intersection in the line of the Knaster-Kuratowski-Mazurkiewicz lemma, some consequences of it and some generalizations of Browder's existence of continuous selection and fixed point theorem are presented.
\end{abstract}

Keywords: Abstract Convexity, Fixed Point, KKM. 


\section{Introduction}

The notion of convexity is a basic mathematical structure used to analyze many different problems. In the literature, many papers have been dealing with the problem of generalizing usual convexity from different points of view. Thus, c-spaces (Horvath [7]), simplicial convexity (Bielawski, [2]), geodesic convexity (Rapcsák, [21]), L-convexity (Ben-El-Mechaiekh et al., [1]) or convexity induced by an order (Horvath and Llinares, [9]) are some of these generalizations.

In general, we can consider two different kinds of generalizations of this notion. On the one hand, those that are motivated by concrete problems, (e.g., the existence of continuous selections and fixed points $[1,2,7,15,18,27]$, or non-convex optimization problems [11, 19, 21, 22, 23], etc.) and, on the other hand, those stated from an axiomatic point of view, where the notion of abstract convexity is based on properties of a family of sets (similar to the properties of the convex sets in topological vector spaces) $[3,17,20,25]$.

In the context of abstract convexity, there are some authors who consider different definitions of abstract convexity asking for additional conditions for the family of subsets which defines the convexity. For instance, Wieczorek [28] considers a convexity on a topological space $X$ as a family of closed subsets $\mathcal{C}$ of $X$ which contains $X$ as an element and which is closed under arbitrary intersections. Note that this definition of abstract convexity does not generalize the notion of usual convexity (in topological vector spaces).

In this paper we consider some abstract convexities that have been used in the literature in order to generalize some results on the existence of continuous selections and fixed points to correspondences. In this framework we focus on an abstract convexity structure called $m c$-spaces (introduced in Llinares $[13,15])$ which is based on the idea of substituting the segment which joins any pair of points (or the convex hull of a finite set of points) for a set which plays their role, and study the relationship between it and simplicial convexity [2], c-spaces [7, 8], L-convexity and B'-simplicial convexity [1] and the convexity induced by an order used in [9]. As an application, we present in the context of mc-spaces a characterization result of non-empty finite intersection in the line of the Knaster Kuratowski Mazurkiewicz lemma (KKM), as well as, an extension of the Browder's result on the existence of continuous selection and fixed point to correspondences with open lower sections.

\section{Abstract convexities}

As we have mentioned before, we are going to present some particular abstract convexities that appear in the literature in relation to the problem 
of the existence of continuous selections and fixed points to correspondences. First we present the general notion of abstract convexity structure.

Definition 1 A family $\mathcal{C}$ of subsets of a set $X$ is an abstract convexity structure for $X$, and the pair $(X, \mathcal{C})$ is a convex space if $\emptyset$ and $X$ belong to $\mathcal{C}, \mathcal{C}$ is closed under arbitrary intersections and under unions of chains.

The elements of $\mathcal{C}$ are called $\mathcal{C}$-convex (or simply abstract convex) subsets of $X$. Moreover, the abstract convexity notion allows us to raise the definition of the convex hull operator, which is similar to that of the closure operator in topology.

Definition 2 If $X$ is a set with an abstract convexity $\mathcal{C}$, and $A$ is a subset of $X$, then the hull operator generated by a convexity structure $\mathcal{C}$, which we will call $\mathcal{C}$-hull (or convex hull) is defined by,

$$
C_{\mathcal{C}}(A)=\cap\{B \in \mathcal{C}: A \subseteq B\}
$$

This operator enjoys certain properties identical to those of usual convexity: for instance, $C_{\mathcal{C}}(A)$ is the smallest $\mathcal{C}$-convex set which contains set $A$.

Although there are more abstract convexities (in connection to the field of fixed point theory) than the ones we are going to present (for instance, Michael's convex structure [16], Komiya convex spaces [12], etc.), most of them are particular cases of c-spaces or simplicial convexity (see Bielawski [2], or Park and Kim [18]) and we will only focus on those that are more intuitive.

\section{$2.1 \quad K$-convex structure}

The $\mathrm{K}$-convex structure is based on the idea of considering functions joining pairs of points. That is, the segments used in usual convexity are substituted for an alternative path previously fixed on $X$. The function that defines this set is called $\mathrm{K}$-convex function and $X$ is said to have a $\mathrm{K}$-convex structure.

Definition 3 (Horvath [6]) A $K$-convex structure on the set $X$ is given by a mapping

$$
K: X \times X \times[0,1] \rightarrow X
$$

Furthermore $(X, K)$ will be called a $K$-convex space.

Note that if $(X, K)$ is a $K$-convex space then it is possible to associate, for any pair of points $x, y \in X$ a subset given by $K(x, y,[0,1])=\cup\{K(x, y, t)$ : $t \in[0,1]\}$ (in a similar way to the case of the union operation, see Prenowitz and Jantosciak [20], or interval spaces, see Stachó [23]). Moreover, we can 
consider a family $\mathcal{C}$ of subsets of $X$, which is an abstract convexity on $X$, as follows,

$$
Z \in \mathcal{C} \Leftrightarrow \forall x, y \in Z \quad K(x, y,[0,1]) \subseteq Z .
$$

The elements of $\mathcal{C}$ will be called K-convex sets.

If continuity as well as other conditions are imposed on function $K$, then particular structures can be defined, where the meaning of this function is completely clear. Now some particular cases of those K-convex structures are presented.

Definition 4 (Llinares, $[13,14]$ ) If $X$ is a topological space, a $\boldsymbol{K}$-convex continuous structure is defined by a continuous function $K: X \times X \times$ $[0,1] \rightarrow X$, such that $K(x, y, 0)=x$, and $K(x, y, 1)=y$.

From function $K$, it is clear that a family of continuous paths joining pairs of points of $X$ can be defined: for any $x, y \in X$,

$$
K_{x y}:[0,1] \rightarrow X, K_{x y}(t)=K(x, y, t)
$$

Obviously a K-convex continuous structure can be defined in any usual convex subset of a topological vector space. Next proposition states conditions on $X$ under which a $\mathrm{K}$-convex continuous structure can be defined.

Proposition 1 If $X$ is a topological space, then it is possible to define a $K$-convex continuous structure on $X$ if, and only if, $X$ is contractible.

Proof. Let $K$ be the function which defines the K-convex continuous structure. Fix $a \in X$, consider the following function,

$$
\begin{aligned}
& H: X \times[0,1] \rightarrow X \\
& H(x, t)=K(x, a, t) .
\end{aligned}
$$

Since $K$ is continuous, then $H$ is a continuous function and, furthermore, it satisfies that $H(x, 0)=x$ and $H(x, 1)=a$, so $X$ is contractible.

Conversely, if $X$ is contractible, then there exists a continuous function $H$ that satisfies the previous assumptions. Then, by considering function $K$ defined as follows,

$$
K(x, y, t)=\left\{\begin{array}{ll}
H(x, 2 t) & t \in\left[0,0^{\prime} 5\right] \\
H(y, 2-2 t) & t \in\left[0^{\prime} 5,1\right]
\end{array},\right.
$$

it is obtained that it defines a $\mathrm{K}$-convex continuous structure on $X$. 
It is important to note that although the contractibility condition and the condition of having a $\mathrm{K}$-convex continuous structure are equivalent, it does not mean that K-convex subsets coincide with contractible subsets. That is due to the fact that the family of contractible sets is not stable under arbitrary intersections, and therefore it does not define an abstract convexity. Hence the abstract convexity defined by function $K$ is given by some of the contractible subsets of $X$ (since it is true that any $\mathrm{K}$-convex set is contractible).

A different case of $\mathrm{K}$-convexity is that of the equiconnected spaces, introduced by Dugundji [4], and Himmelberg [5]. In fact they are a particular case of $\mathrm{K}$-convex continuous spaces.

Definition 5 (Dugundji [4], Himmelberg [5]) A metric topological space $X$ is equiconnected if, and only if, there exists a continuous function $K: X \times X \times[0,1] \rightarrow X$ such that for all $x, y \in X, K(x, y, 0)=x$, $K(x, y, 1)=y, K(x, x, t)=x$ for any $t \in[0,1]$.

In general, absolute retracts spaces ${ }^{1}$ (AR) are equiconnected spaces (Dugundji, [4]). Moreover, in contexts of metric spaces with finite dimensionality, equiconnected spaces coincide with AR ones.

Next some examples of sets where a K-convex continuous structure can be defined are shown.

Example 1 If $E$ is a linear space, a subset $X$ of $E$ is called a star-shaped set if, and only if

$$
\exists x_{0} \in X, \text { such that } t x+(1-t) x_{0} \in X \quad \forall x \in X, \forall t \in[0,1]
$$

In this case, function $K: X \times X \times[0,1] \rightarrow X$ can be given as follows,

$$
K(x, y, t)= \begin{cases}(1-2 t) x+2 t x_{0} & t \in\left[0,0^{\prime} 5\right] \\ (2-2 t) x_{0}+(2 t-1) y & t \in\left[0^{\prime} 5,1\right]\end{cases}
$$

Note that function $K$ on the previous example does not define an equiconnected structure on $\mathrm{X}$ since it does not satisfy $K(x, x, t)=x$ for all $t \in[0,1]$. Following example shows that, in general, star-shaped sets are not equiconnected ones.

Example 2 Let $A \subset \mathbb{R}^{2}$ be the following set,

$$
A=\cup\{(x, x / n), x \in[0,1]: n \in \mathbb{N}\} \cup[0,1] \times\{0\}
$$

It is clear that $A$ is a star-shaped set $\left(x_{0}=(0,0)\right)$ but it is not equiconnected since it is not locally equiconnected (see [4, Theorem 2.4]).

\footnotetext{
${ }^{1}$ A space $Y$ is an absolute retract (AR) whenever $Y$ is metrizable and for any metrizable $X$ and any closed subset $A \subseteq X$, it is verified that each continuous function $f: A \rightarrow Y$ is extendable over $X$.
} 


\subsection{Order convexity}

If $(X, \leq)$ is a partially ordered set (poset), and for all $x, y \in X$ it is denoted by $[x, y]=\{z \in X: x \leq z \leq y\}$ the closed interval, then it is possible to define an abstract convexity structure on $X$ called order convexity, by considering the abstract convex sets as those sets $Z \subseteq X$ such that for all $x, y \in Z,[x, y] \subseteq Z$.

Moreover if $(X, \leq)$ is a (sup)semilattice and it is denoted by $x \vee y$ the supremum of $(x, y)$, then it is possible to consider the abstract convex sets as those subsets $Z \subseteq X$ such that for all $x, y \in Z,[x, x \vee y] \cup[y, x \vee y] \subseteq Z$. In this context, in the paper by Horvath and Llinares [9] is considered that $(X, \leq)$ is a topological semilattice with path-connected intervals and by using the corresponding abstract convexity obtain an order theoretical version of the Knaster-Kuratowski-Mazurkiewicz result as well as fixed point theorems for correspondences.

\section{3 c-spaces}

We can consider abstract convexities on a set $X$ defined by associating a subset of $X$ to any finite family of points of $X$. This subset is in some sense, the generalized convex hull of these points. This is the case, for instance, of the notion of c-space (or H-space) introduced by Horvath [8], that consists of associating an infinitely connected set $\left(C^{\infty}\right)$ satisfying some monotone conditions to any finite subset of $X$. A set $A$ is infinitely connected if every continuous function defined on the boundary of a finite dimensional sphere, with values in $A$ can be extended to a continuous function on the ball, with values in $A$.

Formally, the notion of c-space is as follows,

Definition 6 (Horvath [8]) If $X$ is a topological space and $\langle X\rangle$ denotes the family of non-empty finite subsets of $X$, then a c-structure on $X$ is given by a non-empty set valued map $\Gamma:\langle X\rangle \rightarrow X$ that satisfies:

1. for all $A \in\langle X\rangle, \Gamma(A)$ is non-empty and infinitely connected.

2. for all $A, B \in\langle X\rangle, A \subset B$ implies $\Gamma(A) \subseteq \Gamma(B)$.

The pair $(X, \Gamma)$ is called $c$-space, and a subset $Z \subset X$ is called an $\boldsymbol{H}$-set if, and only if, it is satisfied for all $A \in\langle Z\rangle, \quad \Gamma(A) \subseteq Z$.

Note that this definition includes as a particular case the notion of usual convexity in topological vector spaces. Moreover, it is easy to see that the family of H-sets defines an abstract convexity on $X$.

Remark 1 In Horvath $[6,7]$ a more restrictive notion of c-structure is considered, by assuming that the sets $\Gamma(A)$ are contractible instead of infinitely connected. 


\subsection{Simplicial convexity}

Bielawski [2] introduces an abstract convexity structure from a family of continuous functions. In particular, he associates, to any finite subset of $X$, a continuous function defined on the standard simplex satisfying some conditions.

Definition 7 (Bielawski [2]) If $X$ is a topological space and $\Delta_{k}$ the $k$ dimensional simplex, then $X$ has a simplicial convexity if for each $n \in \mathbb{N}$, and for each $\left(x_{1}, x_{2}, \ldots, x_{n}\right) \in X^{n}$, there exists a continuous function $\Phi\left[x_{1}, x_{2}, \ldots, x_{n}\right]: \Delta_{n-1} \rightarrow X$ that satisfies

1. for all $x \in X, \Phi[x](1)=x$,

2. for all $n \geq 2$, for all $\left(x_{1}, x_{2}, \ldots, x_{n}\right) \in X^{n}$, for all $\left(t_{1}, t_{2}, \ldots, t_{n}\right) \in \Delta_{n-1}$, if $t_{i}=0$, then

$$
\Phi\left[x_{1}, x_{2}, \ldots, x_{n}\right]\left(t_{1}, t_{2}, \ldots, t_{n}\right)=\Phi\left[x_{-i}\right]\left(t_{-i}\right),
$$

where $x_{-i}$ denotes that $x_{i}$ is omitted in $\left(x_{1}, x_{2}, \ldots, x_{n}\right)$.

Moreover a subset $Z$ of $X$ is called a simplicial convex set if, and only if, for all $n \in \mathbb{N}$ and for all $\left(a_{1}, a_{2}, \ldots, a_{n}\right) \in Z^{n}$ it is satisfied that

$$
\text { for all } u \in \Delta_{n-1} \quad \Phi\left[a_{1}, a_{2}, \ldots, a_{n}\right](u) \in Z \text {. }
$$

It is easy to show that simplicial convex sets are stable under arbitrary intersections, therefore they define an abstract convexity structure.

\subsection{B'-simplicial convexity}

The following abstract convexity we present is an obvious generalization of the notion of simplicial convexity (to define it, we only drop condition 1 from definition 7). In this case, a continuous function to every finite subset of elements satisfying only 2 from definition 7 , is associated.

Definition 8 (Ben-El-Mechaiekh et.al. [1]) A topological space X has a B'simplicial convexity if for each $n \in \mathbb{N}$, and for each $\left(x_{1}, x_{2}, \ldots, x_{n}\right) \in X^{n}$, there exists a continuous function $\Phi\left[x_{1}, x_{2}, \ldots, x_{n}\right]: \Delta_{n-1} \rightarrow X$ satisfying that for all $n \geq 2$, for all $\left(x_{1}, x_{2}, \ldots, x_{n}\right) \in X^{n}$, and for all $\left(t_{1}, t_{2}, \ldots, t_{n}\right) \in$ $\Delta_{n-1}$, if $t_{i}=0$, then

$$
\Phi\left[x_{1}, x_{2}, \ldots, x_{n}\right]\left(t_{1}, t_{2}, \ldots, t_{n}\right)=\Phi\left[x_{-i}\right]\left(t_{-i}\right) .
$$

In this context, a subset $Z$ of $X$ is called a B'-simplicial convex set if, and only if, for all $n \in \mathbb{N}$ and for all $\left(a_{1}, a_{2}, \ldots, a_{n}\right) \in Z^{n}$ it is satisfied that

$$
\text { for all } u \in \Delta_{n-1} \quad \Phi\left[a_{1}, a_{2}, \ldots, a_{n}\right](u) \in Z \text {. }
$$


It is obvious that the family of B'-simplicial convex sets is an abstract convexity, and the convex hull induced by this convexity is a subsimplicial hull (notion introduced by Wieczorek, [27]). Moreover, the abstract convex sets obtained from a subsimplicial hull are B'-simplicial convex sets.

\subsection{L-spaces}

Other abstract convexity, which appears in the context of existence of continuous selections and fixed points to correspondences, is the notion of $L$-space (Ben-El-Mechaiekh et al. [1]). This abstract convexity generalizes the (B') simplicial convexity as well as the notion of c-spaces.

Definition 9 (Ben-El-Mechaiekh et al. [1]) An L-structure on $X$ is given by a non-empty set-valued map $\Gamma:\langle X\rangle \rightarrow X$, such that for every $A \in\langle X\rangle$, namely $A=\left\{a_{0}, a_{1}, \ldots, a_{n}\right\}$, there exists a continuous function $f^{A}: \Delta_{n} \rightarrow \Gamma(A)$ such that for all $J \subset\{0,1, \ldots, n\}$, if $\Delta_{J}=C\left\{e_{i_{k}}: i_{k} \in J\right\}$, then $f^{A}\left(\Delta_{J}\right) \subseteq \Gamma\left(\left\{a_{i}: i \in J\right\}\right)$.

The pair $(X, \Gamma)$ is then called $\boldsymbol{L}$-space and a subset $Z$ of $X$, is called an L-convex set if for all $A \in\langle Z\rangle$, then $\Gamma(A) \subset Z$.

Clearly, the family of L-convex sets forms an abstract convexity structure on $X$. Furthermore it is immediate that the notion of $G$-convex spaces used by Park and Kim [18], is a particular case of L-spaces since they require moreover (of definition ??) a monotone condition on the set-valued map $\Gamma$.

\section{7 mc-spaces}

If we go back to the $K$-convex continuous structure, and the continuity condition on function $K$ is relaxed, then we obtain a generalization of this concept. Now the idea is to associate, for any finite set of points, a family of functions requiring their composition to be a continuous function. The image of this composition generates a set, associated with the finite set of points, in a similar way to the case of c-spaces or simplicial convexity. However, in contrast with these cases, no monotone condition on the associated sets is now required.

Definition 10 (Llinares $[13,15])$ A topological space $X$ is an mc-space (or has an mc-structure) if for any non-empty finite subset of $X, A \subset X$, there exists an ordering on it, namely $A=\left\{a_{0}, a_{1}, \ldots, a_{n}\right\}$, a family of elements $\left\{b_{0}, b_{1}, \ldots, b_{n}\right\} \subset X$, and a family of functions $P_{i}^{A}: X \times[0,1] \rightarrow X$, such that for $i=0,1, \ldots, n$,

$$
\text { 1. } P_{i}^{A}(x, 0)=x, P_{i}^{A}(x, 1)=b_{i} \text {, for all } x \in X \text {. }
$$


2. The following function $G_{A}:[0,1]^{n} \rightarrow X$ given by

$$
G_{A}\left(t_{0}, t_{1}, \ldots, t_{n-1}\right)=P_{0}^{A}\left(\ldots\left(P_{n-1}^{A}\left(P_{n}^{A}\left(b_{n}, 1\right), t_{n-1}\right), \ldots, t_{0}\right),\right.
$$

is a continuous function.

Note that the notion of mc-space ranges over a wide field of possibilities, since it can appear in completely different contexts. For instance, if $X$ is a non-empty topological space then it is always possible to define an mcstructure on it by considering, for all $i=0, \ldots,|A|$, the family of functions $P_{i}^{A}$ as follows: by fixing $a \in X,(X \neq \emptyset)$ we consider

1. $P_{i}^{A}(x, t)=x$, for all $x \in X$, for all $t \in[0,1)$,

2. $P_{i}^{A}(x, 1)=a$ for all $x \in X$.

Then, it is clear that from these functions it is obtained that $G_{A}\left(t_{0}, t_{1}, \ldots, t_{n-1}\right)$ is a continuous function.

In the previous case, functions $P_{i}^{A}$ are independently defined of the finite subset $A$ that is considered. However, in other cases, functions $P_{i}^{A}$ can be directly related with elements of $A$, as in the case of usual convex subsets of a topological vector space. Thus, if $X$ is a usual convex subset of a topological vector space, then for any finite subset $A=\left\{a_{0}, a_{1}, \ldots, a_{n}\right\}$ of $X$, we can define functions $P_{i}^{A}(x, t)=(1-t) x+t a_{i}$, which represent the segment that joins $a_{i}$ and $x$ up when $t \in[0,1]$. In this case, the image of function $G_{A}$, coincides with the convex hull of $A$.

Moreover, if $X$ has a K-convex continuous structure and we define functions $P_{i}^{A}(x, t)=K\left(x, a_{i}, t\right)$, then they define an mc-structure on $X$. Therefore mc-spaces are also extensions of $\mathrm{K}$-convex continuous spaces.

Remark 2 Note that if $P_{i}^{A}(x, t)$ is continuous on $t$, then $P_{i}^{A}(x,[0,1])$ represents a continuous path that joins $x$ and $b_{i}$. These paths depend on the points that are considered, as well as on the finite subset $A$ which contains them. Thus, in this case, function $G_{A}$ can be interpreted as follows: $P_{n-1}^{A}\left(b_{n}, t_{n-1}\right)=p_{n-1}$ represents a point of the path that joins $b_{n}$ and $b_{n-1}$, $P_{n-2}^{A}\left(p_{n-1}, t_{n-2}\right)=p_{n-2}$ is a point in the path that joins $p_{n-1}$ and $b_{n-2}$, etc. Therefore, $G_{A}$ can be seen as a composition of these paths and considered as an abstract convex combination of the finite set $A$.

\section{(Insert about here Fig. 1)}

Given an mc-structure, it is possible to define an abstract convexity by considering the family of sets that are stable under function $G_{A}$. In order to define this convexity, we need some previous concepts. 
Definition 11 If $X$ is an mc-space, and $Z$ a subset of $X$, then for all $A \in\langle X\rangle$ such that $A \cap Z \neq \emptyset$, namely $A \cap Z=\left\{a_{i_{0}}, a_{i_{1}}, . ., a_{i_{m}}\right\} \quad\left(i_{0}<i_{1}<\right.$ .. $\left.<i_{m}\right)$, we define the restriction of function $G_{A}$ to $Z$ as follows,

$$
\begin{gathered}
G_{A \mid Z}:[0,1]^{m} \rightarrow X \\
G_{A \mid Z}(t)=P_{i_{0}}^{A}\left(\ldots P_{i_{m-1}}^{A}\left(P_{i_{m}}^{A}\left(a_{i_{m}}, 1\right), t_{i_{m-1}}\right) \ldots, t_{i_{0}}\right),
\end{gathered}
$$

where $P_{i_{k}}^{A}$ are the functions associated with the elements $a_{i_{k}} \in A \cap Z$.

By making use of this notion, now we define mc-sets, which generalize usual convex sets.

Definition $12 A$ subset $Z$ of an mc-space $X$ is an mc-set if, and only if, it is satisfied that for all $A \in\langle X\rangle$, such that $A \cap Z \neq \emptyset$, then

$$
G_{A \mid Z}\left([0,1]^{m}\right) \subseteq Z
$$

where $m=\operatorname{card}(A \cap Z)-1$.

Since the family of mc-sets is stable under arbitrary intersections, it defines an abstract convexity on $X$. Furthermore, we can define the mchull operator in the usual way (denoted by $C_{m c}$ ). So, it is obvious that for all $Z \subset X$, and for all $A \in\langle X\rangle$ such that $A \cap Z \neq \emptyset$, it is satisfied that

$$
G_{A \mid Z}\left([0,1]^{m}\right) \subseteq C_{m c}(Z)
$$

\section{Relation between the different abstract convex- ities}

In this Section the relationship between the different abstract convexities introduced in the previous Section is analyzed. Some of them are easy to prove: for instance, to show that an equiconnected space has a Kconvex continuous structure, or that the $\mathrm{K}$-convex continuous structure is a particular case of the mc-space. Those which are not immediately obtained, are proven throughout this Section.

We start by showing that a K-convex continuous space is a c-space in which the $\mathrm{K}$-convex sets are $\mathrm{H}$-sets.

Proposition 2 If $(X, K)$ is a $K$-convex continuous space, then there exists a non-empty set valued map $\Gamma:\langle X\rangle \rightarrow X$ such that $(X, \Gamma)$ is a c-space. Moreover, $K$-convex sets are $H$-sets. 
Proof. If $(X, K)$ is a $K$-convex continuous space, then we can define the mapping $\Gamma:\langle X\rangle \rightarrow X$, by $\Gamma(A)=C_{K}(A)$ and, by applying Proposition 1 , we know that $\Gamma(A)$ is contractible (and then $C^{\infty}$ ). Moreover, it is immediate to prove that for all $A, B \in\langle X\rangle$, if $A \subset B$ then $\Gamma(A) \subseteq \Gamma(B)$, thus $(X, \Gamma)$ is a c-space. To show that $K$-convex sets are H-sets, assume by contradiction, that there exists a $K$-convex set $Z$, such that $A \in\langle Z\rangle$ and $\Gamma(A)=C_{K}(A) \varsubsetneqq Z$. Then, we have that $A \subset C_{K}(A), A \subset Z$ and both of them are $K$-convex sets, so $A \subset Z \cap C_{K}(A) \varsubsetneqq C_{K}(A)$, which is a contradiction with the fact that $C_{K}(A)$ is the smallest $K$-convex set containing $A$.

Proposition 3 If $(X, K)$ is a topological space with a $K$-convex continuous structure, then it is possible to define a simplicial convexity on $X$. Moreover, $K$-convex sets are simplicial convex sets.

Proof. For any $n \in \mathbb{N}$, and for any $\left(a_{1}, a_{2}, . . a_{n}\right) \in X^{n}$, we define the family of functions $\Phi\left[a_{1}, a_{2}, . . a_{n}\right]$ as follows,

1. if $n=1, \quad \Phi[a]=K(a, a, 1)$,

2. for $n \geq 2$,

$$
\left.\Phi\left[a_{1}, a_{2}, . . a_{n}\right]\left(t_{1}, t_{2}, . ., t_{n}\right)=K\left(\ldots K\left(K\left(a_{n}, a_{n-1}, t_{n-1}\right), a_{n-2}, t_{n-2}\right) \ldots\right), a_{1}, t_{1}\right)
$$

which clearly satisfy conditions from Definition 7 . Moreover, the simplicial convexity generated by $\Phi$ coincides with the one that is obtained from $K$.

Next proposition shows the relationship between order convexity (in topological semilattices) and simplicial convexity.

Proposition 4 If $(X, \leq)$ is a topological (sup)semilattice with path connected intervals, then there exists a simplicial convexity on $X$. Moreover order convex sets are simplicial convex sets.

Proof. If $(X, \leq)$ is a topological (sup)semilattice with path connected intervals, then we can define a non-empty set valued map $\Gamma:\langle X\rangle \rightarrow X$ defined by $\Gamma(A)=\cup_{a \in A}[a, \sup A]$. Moreover, by applying a result of Horvath and Llinares [9, lemma 2.1] we know that for any $n \in \mathbb{N}$, any continuous function $g: \partial \Delta_{n} \rightarrow \Gamma(A)$ can be extended to a continuous function $f: \Delta_{n} \rightarrow \Gamma(A)$, so $\Gamma(A)$ is $C^{\infty}$. Then, if we define $h u l l\{A\}=\Gamma(A)$, the family of order convex sets is an abstract convexity such that hull $\{A\}$ is $C^{\infty}$ and, by applying Bielawski's result [2, Proposition 1.5], we obtain the conclusion.

The relationship between c-spaces and simplicial convexity is shown in the following proposition. 
Proposition 5 (Ben-El-Mechaiekh et al. [1, Corollary 3.7]) If $(X, \Gamma)$ is a c-space such that for all $x \in X, x \in \Gamma(\{x\})$, then $X$ has a simplicial convexity. Moreover $H$-sets are simplicial convex sets.

Next result is immediately obtained from the definition of simplicial convexity.

Proposition 6 If $X$ is a topological space with a simplicial convexity, then this simplicial convexity defines a $B^{\prime}$ 'simplicial convexity. Moreover simplicial convex sets are $B^{\prime}$-simplicial convex sets.

Next proposition establishes the relationship between the notion of cspace and the notion of B'-simplicial convexity.

Proposition 7 (Ben-El-Mechaiekh et al. [1, Proposition 3.6]) If $(X, \Gamma)$ is a c-space, then it is possible to define a B'-simplicial convexity. Moreover $H$-sets are $B$ '-simplicial convex sets.

Next result shows the relationship between B'-simplicial convexity and L-convexity, showing that L-space is more general.

Proposition 8 If $X$ has a $B^{\prime}$-simplicial convexity then $X$ is an $L$-space. Moreover, $B$ '-simplicial convex sets are $L$-convex sets.

Proof. For every $n \in \mathbb{N}$, let $\Omega_{n}$ the set of all functions $\sigma:\{0,1, \ldots, n\} \rightarrow$ $\{0,1, \ldots, n\}$, then for any $A \in\langle X\rangle, A=\left\{a_{0}, a_{1}, \ldots, a_{n}\right\}$ we define the mapping $\Gamma:\langle X\rangle \rightarrow X$ as follows,

$$
\Gamma(A)=\cup\left\{\Phi\left[a_{\sigma(0)}, a_{\sigma(1)}, . . a_{\sigma(n)}\right]\left(\Delta_{n}\right): \sigma \in \Omega_{n}\right\},
$$

and function $f^{A}: \Delta_{n} \rightarrow \Gamma(A)$ by

$$
f^{A}(\lambda)=\Phi\left[a_{0}, a_{1}, . . a_{n}\right](\lambda) \quad \forall \lambda \in \Delta_{n} .
$$

Function $f^{A}$ is continuous and satisfies that for all $J=\left\{i_{0}, \ldots, i_{m}\right\} \subset$ $\{0,1, \ldots, n\},\left(i_{0}<i_{1} \ldots<i_{m}\right)$ and for all $\lambda \in \Delta_{J}$,

$$
\begin{aligned}
f^{A}(\lambda) & =\Phi\left[a_{0}, . . a_{n}\right](\lambda)=\Phi\left[a_{i_{0}}, . ., a_{i_{m}}\right]\left(\lambda_{i_{0}}, . ., \lambda_{i_{m}}\right) \subseteq \\
& \subseteq \Phi\left[a_{i_{0}}, . ., a_{i_{m}}\right]\left(\Delta_{m}\right) \subseteq \Gamma\left(\left\{a_{i_{0}}, . ., a_{i_{m}}\right\}\right),
\end{aligned}
$$

so $f^{A}\left(\Delta_{J}\right) \subseteq \Gamma\left(\left\{a_{i_{0}}, \ldots, a_{i_{m}}\right\}\right)$.

Consider now a B'-simplicial convex set $Z \subseteq X$, since for any $n \in \mathbb{N}$ and any $\left(a_{0}, a_{1}, \ldots, a_{n}\right) \in Z^{n+1}$ we know that $\Phi\left[a_{0}, a_{1}, . . a_{n}\right]\left(\Delta_{n}\right) \subseteq Z$, then for any $A \in\langle Z\rangle, \Gamma(A) \subseteq Z$; therefore $Z$ is an L-convex set. 
Next propositions show that the notion of mc-space and L-space are equivalents in the sense that if we have an mc-space, then it is possible to define an L-structure such that mc-sets are L-sets, and conversely if we have an L-space then, it is possible to define an mc-structure such that L-sets are mc-sets.

Proposition 9 If $X$ is an L-space, then $X$ is an mc-space. Moreover, Lconvex sets are $m c$-sets.

Proof. If $X$ has an L-structure then we can define for all $A \in\langle X\rangle$, $A=\left\{a_{0}, a_{1}, \ldots, a_{n}\right\}$ functions $P_{i}^{A}$ as follows:

$$
\begin{gathered}
P_{n}^{A}\left(a_{n}, 1\right)=f^{A}\left(e_{n}\right), \\
P_{n-1}^{A}\left(P_{n}^{A}\left(a_{n}, 1\right), t_{n-1}\right)=f^{A}\left(t_{n-1} e_{n-1}+\left(1-t_{n-1}\right) e_{n}\right), \\
P_{n-2}^{A}\left(P_{n-1}^{A}\left(P_{n}^{A}\left(a_{n}, 1\right), t_{n-1}\right), t_{n-2}\right)= \\
=f^{A}\left(t_{n-2} e_{n-2}+\left(1-t_{n-2}\right)\left[t_{n-1} e_{n-1}+\left(1-t_{n-1}\right) e_{n}\right]\right),
\end{gathered}
$$

and so on. Moreover, functions $P_{i}^{A}$ are defined in those values not considered until now in such a way that $P_{i}^{A}(x, 0)=x$, and $P_{i}^{A}(x, 1)=f^{A}\left(e_{i}\right)$. Therefore, function

$$
G_{A}\left(t_{0}, t_{1}, \ldots, t_{n-1}\right)=f^{A}\left(\sum_{i=0}^{n} \alpha_{i} e_{i}\right)
$$

where coefficients $\alpha_{i}$ depend continuously on $t_{j}, j=0,1, \ldots, n$. Moreover if $Z$ is a L-convex set, then it is satisfied that for all $A \in\langle Z\rangle, \Gamma(A) \subseteq Z$. To see that $Z$ is also an mc-set, we have to prove that for all $A \in\langle X\rangle$, such that $A \cap Z \neq \emptyset, A \cap Z=\left\{a_{i_{0}}, \ldots, a_{i_{m}}\right\}$, then $G_{A \mid Z}\left([0,1]^{m}\right) \subseteq Z$, so if $J=\left\{i_{0}, \ldots, i_{m}\right\}$ then $\Delta_{J}=C\left(\left\{e_{i_{k}}: k=0, \ldots, m\right\}\right)$ and therefore, by the definition of function $G_{A}$, we get

$$
G_{A \mid Z}\left([0,1]^{m}\right) \subseteq f^{A}\left(\Delta_{J}\right) \subseteq \Gamma\left(\left\{a_{i_{k}}: k=0, \ldots, m\right\}\right)=\Gamma(A \cap Z) \subseteq Z .
$$

To prove the next proposition we use the following lemma, which was proven in Llinares [15, proof of lemma 1].

Lemma 1 (Llinares, [15]) If $X$ is an mc-space, $t_{i}: \Delta_{n} \rightarrow[0,1]$ for $i=0,1, \ldots, n$, functions defined by

$$
t_{i}(\lambda)= \begin{cases}0 & \text { if } \lambda_{i}=0 \\ \frac{\lambda_{i}}{\sum_{j=i}^{n} \lambda_{j}} & \text { if } \lambda_{i} \neq 0\end{cases}
$$

and function $\mathcal{T}: \Delta_{n} \rightarrow[0,1]^{n}$ is defined by $\mathcal{T}(\lambda)=\left(t_{0}(\lambda), t_{1}(\lambda), \ldots, t_{n-1}(\lambda)\right)$, then for any finite set $A \in\langle X\rangle, A=\left\{a_{0}, a_{1}, \ldots, a_{n}\right\}$ the composition $f^{A}=G_{A} \circ \mathcal{T}$ is a continuous function. 
Proposition 10 If $X$ is an mc-space, then $X$ is an L-space. Moreover, mc-sets are L-convex sets.

Proof. If $X$ is an mc-space then, for any finite set $A \in\langle X\rangle, A=$ $\left\{a_{0}, a_{1}, \ldots, a_{n}\right\}$ there exists a family of functions $P_{i}^{A}$, such that their composition $G_{A}$ is a continuous function. Then from lemma 1 the function $f^{A}: \Delta_{n} \rightarrow X$ defined by $f^{A}=G_{A} \circ \mathcal{T}$ is continuous.

To obtain an L-convexity in such a way that mc-sets are L-convex sets, we define the non-empty set valued map $\Gamma:\langle X\rangle \rightarrow X$, as follows,

$$
\Gamma(B)=\cup\left\{G_{A \mid B}\left([0,1]^{m}\right): B \subseteq A, A \in\langle X\rangle\right\},
$$

where $m=\operatorname{card}(A \cap B)-1$.

Notice that for any $A \in\langle X\rangle, A=\left\{a_{0}, a_{1}, \ldots, a_{n}\right\}$, for all $J=$ $\left\{i_{0}, \ldots, i_{m}\right\} \subseteq\{0,1, \ldots, n\},\left(i_{0}<i_{1} \ldots<i_{m}\right)$, and for all $\lambda \in \Delta_{J}$,

$$
f^{A}(\lambda)=G_{A}(\mathcal{T}(\lambda)) \in G_{A \mid\left\{a_{i_{0}}, . ., a_{i_{m}}\right\}}\left([0,1]^{m}\right) \subseteq \Gamma\left(\left\{a_{i_{k}}: i_{k} \in J\right\}\right),
$$

so $f^{A}\left(\Delta_{J}\right) \subseteq \Gamma\left(\left\{a_{i_{0}}, . ., a_{i_{m}}\right\}\right)$.

Moreover, if $Z$ is an mc-set, then for all $A \in\langle X\rangle$, such that $A \cap Z \neq \emptyset$, $A \cap Z=\left\{a_{i_{0}}, \ldots, a_{i_{m}}\right\}$, then $G_{A \mid Z}\left([0,1]^{m}\right) \subseteq Z$. Therefore, for any $B \in\langle Z\rangle$, it is satisfied that $\Gamma(B) \subseteq Z$ by construction of mapping $\Gamma$, so $Z$ is an L-convex set.

The following example shows an mc-space which is not a c-space, in the sense that mc-sets do not coincide with $\mathrm{H}$-sets.

Example 3 Consider the following subset of $\mathbb{R}: X=\bigcup_{n=0}^{\infty}[2 n, 2 n+1]$. Then it is possible to prove that $X$ is an mc-space whose mc-sets are not $\mathrm{H}$-sets. To do it, we define the following functions for all $A=\left\{a_{1}, \ldots, a_{n}\right\} \in\langle X\rangle$

$$
\begin{aligned}
& P_{i}^{A}(x, 0)=x, \\
& P_{i}^{A}(x, t)=\max \left\{a_{i}: a_{i} \in A\right\}=a^{*} \quad \forall t \in(0,1] .
\end{aligned}
$$

It is clear that $G_{A}$ is a continuous function since

$$
G_{A}\left(t_{0}, t_{1}, \ldots, t_{n-1}\right)=P_{0}^{A}\left(\ldots P_{n-1}^{A}\left(P_{n}^{A}\left(a_{n}, 1\right), t_{n-1}\right) \ldots, t_{0}\right)=a^{*}, \forall t_{i} \in[0,1],
$$

therefore $X$ is an mc-space. Moreover, for all $w \in X$, subsets $Z_{w}=$ $[w,+\infty) \cap X$ are mc-sets, since for every finite subset $A$ of $X$ such that $A \cap Z_{w} \neq \emptyset$ we know that $a^{*} \in Z_{w}$, therefore

$$
G_{A \mid Z_{w}}\left([0,1]^{m}\right)=a^{*} \in Z_{w} .
$$

However, it is not possible to define a c-structure on $X$ such that $Z_{w}$ are $\mathrm{H}$-sets for all $w \in X$. If by contradiction we assume that $\Gamma:\langle X\rangle \rightarrow X$ 
defines a c-structure on $X$, then it has to be verified that for all $A \in\langle X\rangle$, $\Gamma(A)$ has to be a contractible set and, therefore, to be included in some interval $[2 n, 2 n+1]$. Moreover, by the monotonicity condition (if $A \subset B$, then $\Gamma(A) \subseteq \Gamma(B))$ this interval has to be the same for every $A \in\langle X\rangle$, since, if not, they belong to two different connected components and they would not be contractible sets. Therefore it is clear now that $Z_{w}$ is not an $\mathrm{H}$-set whenever $w>2 n+1$ (for every $A \in\left\langle Z_{w}\right\rangle$ it is verified $\Gamma(A)$ is not included in $Z_{w}$ ).

Notice that this example is also valid in order to see that in general a mc-structure does not induce a B'-simplicial convexity.

We finish this section by summing up the different relationships between the different abstract convexities in the following diagram.

\section{Insert here Fig. 2}

\section{Selections, fixed point and KKM results}

This section is devoted to presenting some results on the existence of fixed point, continuous selection to correspondences and KKM results in the context of mc-spaces. First result states the existence of a continuous selection, with a fixed point, of the mc-hull of a correspondence defined on mc-spaces. Moreover, it gives the key to obtain the generalization of Browder's result on the existence of continuous selection and fixed point to correspondences with open lower sections. Henceforth we consider Hausdorff topological spaces.

Theorem 1 (Llinares [15]) If $X$ is a compact topological mc-space and $\phi: X \rightarrow X$ is a non-empty valued correspondence satisfying that

$$
y \in \phi^{-1}(x) \Rightarrow \exists x^{\prime} \in X: y \in \operatorname{int} \phi^{-1}\left(x^{\prime}\right),
$$

then, there exists a non-empty finite subset. $A$ of $X$, and a continuous function $f: X \rightarrow X$ such that

1. for all $x \in X, f(x) \in G_{A \mid \phi(x)}\left([0,1]^{m}\right)$;

2. there exists $x^{*} \in X$ such that $x^{*}=f\left(x^{*}\right)$.

Next result is an extension of Browder's theorem to the context of mcspaces.

Theorem 2 If $X$ is a compact topological mc-space and $\phi: X \rightarrow X$ is a non-empty mc-set valued correspondence satisfying one of the following conditions, 
(i) $y \in \phi^{-1}(x) \Rightarrow \exists x^{\prime} \in X: y \in \operatorname{int} \phi^{-1}\left(x^{\prime}\right)$,

(ii) for each $y \in X, \phi^{-1}(y)$ contains an open subset $O_{y}$ of $X$ such that $\cup_{y \in X} O_{y}=X$

then $\phi$ has a continuous selection and a fixed point.

Proof. It is easy to show that conditions (i) and (ii) are equivalent if $\phi$ is a non-empty set valued correspondence, so the conclusion is obtained by applying Theorem 1 and by considering that $\phi$ has mc-set values.

In addition if $\phi$ is a non-empty mc-set valued correspondence with open lower sections, then we can apply this theorem and ensure the existence of a continuous selection and a fixed point.

Furthermore, if paracompacity on the space $X$ is considered instead of compacity, and $X$ has a $B^{\prime}$-simplicial convexity, then we can also ensure the existence of a continuous selection to correspondences with open lower sections.

Theorem 3 (Ben-El-Mechaiekh et al. [1, Proposition 3.8]) If $X$ is a paracompact topological space, $Y$ is a topological space with a $B^{\prime}$-simplicial convexity, $\phi: X \rightarrow Y$ a correspondence with non-empty $B$ '-simplicial convex values and open lower sections, then $\phi$ has a continuous selection.

Next the notion of compressibility (Wieczorek [27]) is presented. This notion is related with results on the existence of continuous selections to correspondences with open lower sections, in spaces with an abstract convexity structure.

Definition 13 (Wieczorek [27]) A topological space $X$ is compressible into a topological space $Y$ (with respect to an operation of hull defined on $Y$ ) if for every finite family $\mathcal{A}$ of open sets covering $X$ and every system $\left\{y_{A}: A \in \mathcal{A}\right\}$ of elements of $Y$, there exists a continuous function $f: X \rightarrow Y$ such that for every $x \in X, f(x) \in C\left(\left\{y_{A}: x \in A\right\}\right)$.

From this definition it is easy to show the following theorem.

Theorem 4 A normal topological space is compressible into

(a) every L-space,

(b) every mc-space. 
Notice the previous Theorems $1,2,3$, could not be obtained as a consequence the results of Wieczorek [27, theorem 8 and corollary 9] since we use weaker conditions. Moreover, we obtain also the existence of fixed points (in theorem 2).

Next some generalizations of the well known Knaster-KuratowskiMazurkiewicz result (KKM) in the contexts of mc-spaces are presented. First we show a characterization result of non-empty finite intersection that contains, as a corollary, the classical KKM-lemma as well as his version in the context of mc-spaces and therefore in all of the abstract convexity structures that we have introduced.

Theorem 5 If $X$ is a topological space and $\left\{R_{i}: i=0, \ldots, n\right\}$ a family of closed subsets of $X$, then the following statements are equivalent:

(i) $\stackrel{\cap}{i=0}_{n}^{n} R_{i} \neq \emptyset$;

(ii) $X$ is an mc-space and there exists a finite set $A=\left\{x_{0}, \ldots, x_{n}\right\} \in\langle X\rangle$ such that for any family $\left\{i_{0}, \ldots, i_{k}\right\} \subseteq\{0,1, \ldots n\}$ of indices, it is satisfied that

$$
G_{A \mid\left\{x_{i_{0}}, \ldots, x_{i_{k}}\right\}}\left([0,1]^{k}\right) \subseteq \bigcup_{j=0}^{k} R_{i_{j}}
$$

Proof. On the one hand, if $X$ is an mc-space we know that for all $A=$ $\left\{x_{0}, \ldots, x_{n}\right\} \in\langle X\rangle$ there exist functions $P_{i}^{A}$ such that their composition, $G_{A}$ is continuous. Moreover, from lemma 1, the function $f=G_{A} \circ \mathcal{T}: \Delta_{n} \rightarrow X$ is continuous. Furthermore if $J=\left\{i_{0}, \ldots, i_{k}\right\} \subseteq\{0, \ldots, n\}$, then by definition of $\mathcal{T}$ and $G_{A}$, we have that

$$
f\left(\Delta_{J}\right) \subseteq G_{A \mid\left\{x_{i}: i \in J\right\}}\left([0,1]^{k}\right) \subseteq \cup_{i \in J} R_{i} .
$$

Therefore $f^{-1}\left(R_{i}\right)=F_{i}$ are closed subsets of $\Delta_{n}$ and it is satisfied that $\Delta_{J} \subseteq \cup_{i \in J} F_{i}$, so we can apply the KKM-lemma and obtain that there exists $z \in \bigcap_{i=0}^{n} F_{i}$, so $f(z) \in \bigcap_{i=0}^{n} R_{i}$.

On the other hand, consider $x^{*} \in \bigcap_{i=0}^{n} R_{i}$, and define the mc-structure in the following way: for all $A \in\langle X\rangle$,

(i) $P_{i}^{A}(x, t)=x, \forall x \in X, t \in[0,1)$ and

(ii) $P_{i}^{A}(x, 1)=x^{*}$,

in this case, $G_{A}\left(t_{0}, \ldots, t_{n-1}\right)=x^{*}$ is continuous and trivially satisfies that for every subfamily $\left\{i_{0}, \ldots, i_{k}\right\} \subseteq\{0, \ldots, n\}$ of indices

$$
G_{A \mid\left\{x_{i_{0}}, \ldots, x_{i_{k}}\right\}}\left([0,1]^{k}\right)=x^{*} \in \bigcup_{j=0}^{k} R_{i_{j}} .
$$


In the same line as in the previous characterization, we obtain the generalization to the non-finite case.

Theorem 6 If $X$ is a topological space and $\phi: X \rightarrow X$ is a non-empty valued correspondence with closed values, and there exists $x_{0} \in X$ such that $\phi\left(x_{0}\right)$ is compact, then the following statements are equivalent:

(i) $\bigcap_{x \in X} \phi(x) \neq \emptyset$;

(ii) $X$ is an mc-space such that for all finite subset $A=\left\{x_{0}, \ldots, x_{n}\right\}$ of $X$, it is satisfied that for any family $\left\{i_{0}, \ldots, i_{k}\right\} \subseteq\{0,1, \ldots n\}$ of indices, then

$$
G_{A \mid\left\{x_{i_{0}}, \ldots, x_{i_{k}}\right\}}\left([0,1]^{k}\right) \subseteq \bigcup_{j=0}^{k} \phi\left(x_{i_{j}}\right) .
$$

As a corollary we obtain the finite version of KKM-lemma in the context of mc-spaces.

Corollary 1 If $X$ is an mc-space, $\left\{R_{i}: i=0, \ldots, n\right\}$ a family of closed subsets of $X$ and there exists points $x_{0}, \ldots, x_{n}$ of $X$ such that for every family $\left\{i_{0}, \ldots, i_{k}\right\} \subseteq\{0,1, \ldots n\}$ of indices

$$
C_{m c}\left(\left\{x_{i_{0}}, \ldots, x_{i_{k}}\right\} \subseteq \bigcup_{j=0}^{k} R_{i_{j}},\right.
$$

then $\bigcap_{i=0}^{n} R_{i} \neq \emptyset$.

To obtain other generalizations of the KKM-lemma, we use the notion of KKM-relation introduced by Wieczorek [27].

Definition 14 (Wieczorek [27]) Given topological spaces $X$ and $Y, Y$ with an abstract convexity structure, a correspondence $\phi: X \rightarrow Y$ is a KKMrelation on a set $F=\left\{x_{1}, \ldots, x_{n}\right\} \subset X$ with respect to a correspondence $\Theta: X \rightarrow Y$ whenever, for every $y_{1} \in \Theta\left(x_{1}\right), \ldots, y_{n} \in \Theta\left(x_{n}\right)$,

$$
C\left(\left\{y_{1}, \ldots, y_{n}\right\}\right) \subseteq \bigcup_{i=1}^{n} \phi\left(x_{i}\right) .
$$

It is said that $\phi$ is a KKM-relation with respect to $\Theta$ if it is a KKM-relation on every finite set $F$.

Corollary 1 can be extended by considering the notion of KKM-relation.

Corollary 2 If $X$ is an mc-space and $\phi: X \rightarrow X$ is a KKM-relation with respect to identity function $(\Theta(x)=x)$ such that has non-empty closed values, then the family $\{\phi(x): x \in X\}$ has the finite intersection property. 
As an immediate consequence we obtain.

Corollary 3 If $X$ is an mc-space and $\phi: X \rightarrow X$ is a KKM-relation with respect to identity function $(\Theta(x)=x)$ such that has non-empty closed values and if there is $x_{0}$ such that $\phi\left(x_{0}\right)$ is compact, then $\underset{x \in X}{\cap} \phi(x) \neq \emptyset$.

In the following theorem we use similar continuity conditions to those considered by Tarafdar [24]. The generalization is as follows,

Theorem 7 If $X$ is a compact mc-space and $\phi: X \rightarrow X$ is a KKM-relation with respect to identity function $(\Theta(x)=x)$ such that for all $x \in X$ the set $X-\phi(x)$ contains an open subset $O_{x}$ that satisfies $\underset{x \in X}{\cup} O_{x}=X$ if $\bigcap_{x \in X} \phi(x)=\emptyset$, then $\bigcap_{x \in X} \phi(x) \neq \emptyset$.

Proof. By contradiction, assume that $\bigcap_{x \in X} \phi(x)=\emptyset$, that is,

$$
X=X-\bigcap_{x \in X} \phi(x)=\bigcup_{x \in X}(X-\phi(x)) .
$$

Since $X=\bigcup_{x \in X} O_{x}$ and $X$ is compact, then there exists a finite subcovering and a finite partition of unity $\left\{\psi_{i}\right\}_{i=0}^{n}$ subordinated to it, that is,

$$
\psi_{i}(x)>0 \text { if, and only if, } x \in O_{a_{i}} \subset X-\phi\left(a_{i}\right), i=0,1, \ldots, n \text {. }
$$

and we define function $\Psi: X \rightarrow \Delta_{n}$ by $\Psi(x)=\left(\psi_{0}(x), \ldots, \psi_{n}(x)\right)$.

Moreover, if we take $A=\left\{a_{0}, \ldots, a_{n}\right\}$, since $X$ is an mc-space, there exists a continuous function $G_{A}:[0,1]^{n} \rightarrow X$, and by applying lemma 1 then, the following function

$$
f: X \stackrel{\Psi}{\rightarrow} \Delta_{n} \stackrel{T}{\rightarrow}[0,1]^{n} \stackrel{G_{A}}{\rightarrow} X,
$$

is continuous. Furthermore, if we consider $g=G_{A} \circ \mathcal{T}$, then function $\Psi \circ g: \Delta_{n} \rightarrow \Delta_{n}$ is a continuous one, defined from a compact convex set into itself, therefore we can apply Brouwer's fixed point theorem and conclude that there exists a fixed point, that is,

$$
\exists y^{*} \in \Delta_{n}: \Psi\left(g\left(y^{*}\right)\right)=y^{*}
$$

and by denoting $x^{*}=g\left(y^{*}\right)$ we obtain that $f\left(x^{*}\right)=x^{*}$.

Therefore if we define $J\left(x^{*}\right)=\left\{i: \psi_{i}\left(x^{*}\right)>0\right\}$, it is satisfied that

$$
x^{*}=G_{A}\left(\mathcal{T}\left(\Psi\left(x^{*}\right)\right)\right) \in C_{m c}\left(\left\{a_{i}: i \in J\left(x^{*}\right)\right\}\right) \subseteq \underset{i \in J\left(x^{*}\right)}{\bigcup} \phi\left(a_{i}\right) .
$$

Furthermore, if $i \in J\left(x^{*}\right)$, then $x^{*} \in O_{a_{i}} \subset X-\phi\left(a_{i}\right)$, hence

$$
x^{*} \in \underset{i \in J\left(x^{*}\right)}{\bigcap}\left(X-\phi\left(a_{i}\right)\right)=X-\left(\underset{i \in J\left(x^{*}\right)}{\cup} \phi\left(a_{i}\right)\right),
$$


which is a contradiction.

Next result is in the line of those of Wieczorek [27], but by considering mc-spaces.

Theorem 8 If $X$ is a normal mc-space, $\left\{x_{0}, x_{1} \ldots, x_{n}\right\} \subset X$ and $\phi: X \rightarrow X$, and $\Theta: X \rightarrow X$ are two correspondences satisfying

(i) $\Theta: X \rightarrow X$, is upper semicontinuous (u.s.c.), such that for all $x \in X, \quad x \in \Theta(x)$

(ii) $\phi: X \rightarrow X$, is a KKM-relation, with respect to $\Theta$,

(iii) for every $x_{*} \in X,\left\{\bigcap_{i=0}^{n} \overline{\phi\left(x_{i}\right)}\right\} \cap \Theta\left(x_{*}\right)=\emptyset$ implies that $\overline{\phi\left(x_{j}\right)} \cap \Theta\left(x_{*}\right)=$ $\emptyset$ for some $j$,

then there exists $x^{\prime} \in X$ such that

$$
\left\{\bigcap_{i=0}^{n} \overline{\phi\left(x_{i}\right)}\right\} \cap \Theta\left(x^{\prime}\right) \neq \emptyset .
$$

In particular if $\overline{\phi\left(x^{*}\right)}$ is compact for some $x^{*} \in X$, then

$$
\bigcap_{x \in X} \overline{\phi(x)} \neq \emptyset \text {. }
$$

Proof. Suppose that for every $x \in X$

$$
\left\{\bigcap_{i=0}^{n} \overline{\phi\left(x_{i}\right)}\right\} \cap \Theta(x)=\emptyset \text {. }
$$

By (iii), we know that for every $x \in X$, there exists some $j$ such that $\overline{\phi\left(x_{j}\right)} \cap \Theta(x)=\emptyset$. Therefore, by the u.s.c. of $\Theta$, the sets $X_{i}=$ $\left\{x: \overline{\phi\left(x_{i}\right)} \cap \Theta(x)=\emptyset\right\}$, for $i=0,1, \ldots, n$, are open and form an open covering of $X$. But since $X$ is a normal space, we know that there exists a partition of unity $\left\{\psi_{i}\right\}_{i=0}^{n}$ subordinated to this covering, so

$$
\psi_{i}(x)>0 \text { if, and only if, } x \in X_{i}, i=0,1, \ldots, n,
$$

and we can define function $\Psi: X \rightarrow \Delta_{n}$ by $\Psi(x)=\left(\psi_{0}(x), \ldots, \psi_{n}(x)\right)$. Moreover, if we choose $y_{0} \in \Theta\left(x_{0}\right), \ldots, y_{n} \in \Theta\left(x_{n}\right)$, and take $A=\left\{y_{0}, \ldots, y_{n}\right\}$, since $X$ is an mc-space, then there exists a continuous function $G_{A}:[0,1]^{n} \rightarrow$ $X$. Therefore from lemma 1 the function $f=G_{A} \circ \mathcal{T} \circ \Psi$,

$$
f: X \stackrel{\Psi}{\rightarrow} \Delta_{n} \stackrel{\tau}{\rightarrow}[0,1]^{n} \stackrel{G_{A}}{\rightarrow} X
$$


is continuous. On the one hand, by reasoning as in the previous theorem, we can conclude that there exists a fixed point of function $f$, that is,

$$
f(w)=w .
$$

On the other hand, this element $w$ satisfies that

$$
\begin{gathered}
w=f(w)=G_{A}(\mathcal{T}(\Psi(w))) \in C_{m c}\left(\left\{y_{i}: w \in X_{i}\right\}\right)= \\
C_{m c}\left(\left\{y_{i}: \overline{\phi\left(x_{i}\right)} \cap \Theta(w)=\emptyset\right\}\right) \subseteq C_{m c}\left(\left\{y_{i}: \phi\left(x_{i}\right) \cap \Theta(w)=\emptyset\right\}\right.
\end{gathered}
$$

and from the KKM-relation condition we obtain

$$
C_{m c}\left(\left\{y_{i}: \phi\left(x_{i}\right) \cap \Theta(w)=\emptyset\right\} \subseteq \cup\left\{\phi\left(x_{i}\right): \phi\left(x_{i}\right) \cap \Theta(w)=\emptyset\right\},\right.
$$

which is a contradiction with the condition that correspondence $\Theta$ contains the diagonal. 


\section{References}

[1] H. Ben-El-Mechaiekh, S. Chebbi, M. Florenzano and J.V. Llinares, Abstract Convexity and Fixed Points, To appear in Journal of Mathematical Analysis and Applications, (1997).

[2] R. Bielawski, Simplicial Convexity and its Applications. Journal of Mathematical Analysis and Applications 127(1987), 155-71.

[3] W.A. Coppel, Axioms for convexity. Bull. Austral. Math. Soc. 47 (1993), 179-197.

[4] J. Dugundji, Locally equiconnected spaces and absolute neighborhood retracts. Fundamenta Mathematicae LVII (1965), 187-93

[5] Ch.J. Himmelberg, Some Theorems on equiconnected and locally equiconnected spaces. Trans. Amer. Math. Soc. 115 (1965), 43-53.

[6] Ch.D. Horvath, Convexité généralisée et applications, in "Méthodes topologiques en analyse convexe", Séminaire de Mathematiques superieures, séminaire scientifique OTAN, 1990.

[7] Ch.D. Horvath, Contractibility and generalized convexity, Journal of Mathematical Analysis and Applications. 156 (1991), 341-357.

[8] Ch.D. Horvath, Extension and selection theorems in topological spaces with a generalized convexity structure, Annales de la Faculté des Sciences de Toulouse. Vol. II, $\mathrm{n}^{0} 2$ (1993), 253-269.

[9] Ch.D. Horvath and J.V. Llinares, Maximal Elements and Fixed Points for Binary Relations on Topological Ordered Spaces. Journal of Mathematical Economics 25 (1996), 291-306.

[10] D.C. Kay and E.W. Womble, Axiomatic convexity theory and relationships between the Carathéodory, Helly and Radon numbers. Pacific Journal of Mathematics 38, $\mathrm{n}^{0} 2$ (1971), 471-485.

[11] J. Kindler and R. Trost, Minimax theorems for interval spaces. Acta Math. Hung. 54 (1-2) (1989), 34-49.

[12] H. Komiya, Convexity on a topological space. Fund. Math. 111 (1981), 107-113.

[13] J.V. Llinares, "Abstract Convexity, Fixed Points and Applications", Ph.D. Thesis, University of Alicante, Spain, 1994.

[14] J.V. Llinares, Existence of maximal elements in a binary relation relaxing the convexity condition. WP-AD 95-10 University of Alicante, Spain, 1995. 
[15] J.V. Llinares, Unified treatment of the problem of the existence of maximal elements in binary relations. A characterization. To appear in Journal Mathematical Economics (1997).

[16] E. Michael, Convex structures and continuous selections. Cand. J. Math. 11 (1959), 556-575.

[17] L. Nachbin, On the convexity of sets. Academy of sciences Mathematics $40, \mathrm{n}^{\circ} 3(1992)$.

[18] S. Park and H. Kim, Admissible classes of multifunctions on generalized convex spaces. Proc. Coll. Natur. Sci., SNU, 18, n ${ }^{0}$ 1-2 (1993), 1-21.

[19] R. Pini and C. Singh, A survey of recent [1985-1995] advances in generalized convexity with applications to duality theory and optimality conditions. Optimization 39 (1997), 311-360.

[20] W. Prenowitz and J. Jantosciak, "Join Geometries", Springer Verlag, 1979.

[21] T. Rapcsák, Geodesic convexity in nonlinear optimization. Journal of Optimization Theory and Applications 69, $\mathrm{n}^{0} 1$ (1991), 169-183.

[22] I. Singer, "Abstract convex analysis", Canadian Mathematical Society series of monographs and advanced texts. John Wiley and sons, inc., 1997.

[23] L.L. Stachó, Minimax theorems beyond topological vector spaces. Acta Sci. Math., 42 (1980), 157-164.

[24] E. Tarafdar, Fixed point theorems in H-spaces and equilibrium points of abstract economies, J. Austral. Math. Soc. (Series A), 53 (1992), $252-260$.

[25] M.L.J. Van de Vel, "Theory of Convex Structures", North Holland, Elsevier Science Publishers B.V., 1993.

[26] A. Wieczorek, Fixed Points of Multifunctions in General Convexity Spaces. Report 508 Institute of computer Science, Polish Academy of Sciences, Warsaw (1983).

[27] A. Wieczorek, Compressibility: A property of topological spaces related to abstract convexity. Journal of Mathematical Analysis and Applications 161 (1991), N 1, 9-19.

[28] A. Wieczorek, The Kakutani Property and the Fixed Point Property of Topological Spaces with abstract Convexity. Journal of Mathematical Analysis and Applications 168 (1992), N 2, 483-499. 